Chapter 9

\title{
Does Eradication of Helicobacter pylori Decreases the Expression of p53 and c-Myc oncogenes in the Human Gastric Mucosa?
}

\author{
Hanan AlSaeid Alshenawy and \\ Amr Mahrous Alshafey \\ Additional information is available at the end of the chapter
}

http://dx.doi.org/10.5772/53673

\section{Introduction}

Helicobacter pylori (H.pylori) may cause many gastroduodenal diseases including acute gastritis, atrophic gastritis, intestinal metaplasia, peptic ulcer and others disorders. Although many studies revealed close association between gastric cancer and H.pylori, there have been only few studies that report on gastric carcinogenesis associated with chronic H.pylori $[1,2]$

H.pylori is a class 1 gastric carcinogen. However, it remains unclear whether H.pylori affects molecular alterations in chronic gastritis. It is well known that only a minority of H.pylori positive patients with chronic gastritis develop gastric cancer, and so the link is unclear [2].

Nardone et al [3] had stated that gastric carcinogenesis is a multistep process progressing from chronic gastritis through glandular atrophy, metaplasia, and dysplasia. Acquired genomic instability, generally precedes neoplastic clonal expansion. H.pylori damages stimulates gastric cell proliferation, which leads to mucosal repair, but which can also induce cellular DNA damage. The most frequent epiphenomenon of DNA alteration is activation of oncogenes and/or mutation of oncosuppressor genes. The role of these genes has been studied in gastric carcinogenesis, but their interrelation with H.pylori infection has yet to be defined. Thus, this study was designed to detect the relation between H.pylori and gastric carcinogenesis and to investigate the effect of its eradication on the expression of p53 and cMyc in H.pylori associated chronic gastritis.

As a transcription factor, p53 has roles in regulation of proliferation, apoptosis, genomic repair, controls the onset of cellular senescence and suppresses angiogenesis $[4,5]$. p53 muta- 
tion is among the major episodes in the multi-step process of gastric carcinogenesis, while it has also been reported in pre-malignant lesions of the stomach, such as chronic atrophic gastritis, intestinal metaplasia, and dysplasia [1].

c-Myc is a basic transcriptional factor that regulates several genes involved in cell proliferation, differentiation, apoptosis, and oncogenesis. c-Myc expression was studied in gastric cancer with H.pylori infection by many authors [6,7]. Also the relationship between H.pylori infection and c-Myc expressions was investigated in a series of diseases and concluded that in gastric carcinogenesis, H.pylori might cause the imbalance of proliferation and apoptosis in precancerous lesions, leading to tumor-suppressor system mutation and telomerase reactivation, and finally causes gastric cancer [8].

\section{Material and methods}

\subsection{Patients and tissue collection}

A total of 55 chronic gastritis patients (38 male and 17 female with mean age: 57.1 ) underwent endoscopic examination for evaluation of dyspypsia. H.pylori-positive $(+)$ were 45 cases while H.pylori-negative (-) were only 10 cases. All H.pylori-positive patients had successful eradication therapy (15 days of treatment with omeprazole $20 \mathrm{mg}$ twice a day, clarithromycin $500 \mathrm{mg}$ twice a day, and tinidazole $500 \mathrm{mg}$ twice a day), and underwent biopsies before and six months after eradication.

\subsection{Detection of $H$. pylori in gastric biopsy specimens}

H.pylori in the stomach was detected by rapid urease test, and histological examination. For the urease test, biopsy specimens were immediately inserted into the rapid urease test solution. H.pylori was histologically detected by May-Giemsa stain. H.pylori eradication was considered successful when the results of the two tests (both the urase test and Giemsa stain) were found negative.

\subsection{Histological evaluation}

Biopsy specimens were taken from five points of the stomach, as recommended by the updated Sydney system [9], i.e. the lesser curvature of the antrum, and the greater curvature of the antrum, the smaller curvature of the angle, and the lesser curvature of the middle corpus, and the greater curvature of the upper corpus. All biopsy materials were fixed in buffered formalin for 24 hours and embedded in paraffin. Serial sections were stained with haematoxylin-eosin and May-Giemsa stain. The status of the gastric mucosa was evaluated according to the updated Sydney system. The degree of inflammation (manifested by mild lymphocyte and plasma-cell infiltration), neutrophil activity, atrophy (glandular morphology absent in the mucosa and replaced by connective tissue, inter-glandular space was infiltrated by plasma cells and lymphocytes), dysplasia (nuclear atypia with or without architectural abnormalities in the gastric epithelium, but without invasion) and intestinal 
metaplasia (presence of goblet cells in gastric mucosa) were classed by four grades, with 0 being for 'normal', 1 for 'mild', 2 for 'moderate', and 3 for 'marked'.

\subsection{Immunohistochemical detection of p53and c-Myc}

Serial paraffin sections were washed in $1 / 15 \mathrm{~mol} / \mathrm{L}$ phosphate buffered saline $(\mathrm{pH} 7.4)$ three times for five minutes, and pre-incubated in normal rabbit serum (1:10 in PBS) for 20 min. Next, sections were incubated with primary antibodies for 16 hours at 4 C, followed by the avidin-biotin complex method. The sections were immersed in $0.05 \mathrm{~mol} / \mathrm{L}$ Tris- $\mathrm{HCl}$ buffer containing $0.02 \% 3$, 3'-diaminobenzidine tetrahydrochloride and $0.005 \% \mathrm{H}_{2} \mathrm{O}_{2}$, and the nuclei were counterstained with hematoxylin. Control sections incubated with normal mouse IgG instead of the primary antibody showed no non-specific staining. The primary antibodies used in this study for p53, staining the sections were incubated with anti-rabbit or mouse antibody (Envision HRP, Dako Company) for $10 \mathrm{~min}$ and anti-c-Myc protein (Oncogene Science, San Diego, California, USA; c-Myc p62, dilution 1:50) After antigen retrieval (for c-Myc in 1mM EDTA ( $\mathrm{pH} 8.0$ ), sections were incubated overnight at $41 \mathrm{C}$ with monoclonal mouse antibodies to c-Myc (1:100 dilution, Abcam Inc., Cambridge, MA, USA). Immunoreactivity was detected using the DAB Map Kit (PIERCE, Woburn, MA, USA), based on the avidinbiotin complex immunoperoxidase technique.

\subsection{Evaluation of p53 and c-Myc staining}

The degree of immunopositivity was evaluated semiquantitatively. A total of 300 cells was counted in random fields from representative areas of the lesions, and the immunoreactive cells were roughly assessed and expressed as percentages. The scoring system for both antibodies tested was: 0-5\% (negative -); $5-25 \%$ (low positivity + ); $25-50 \%$ (moderate positivity + + ); $>50 \%$ (high positivity +++) according to Nardone et al [3].

\subsection{Statistical analysis}

All data were put into SPSS 10.0 statistical computer system. Statistical analysis was performed with $t$ test or chi-square test, with statistical significance indicated by a value of $P \leq 0.05$.

\section{Results}

The study included 55 cases, 45 cases of them were H.pylori (+). The neutrophilic infiltrate was detected only in H.pylori (+) cases and the majority of cases were grade 3, 26 cases were positive for lymphoplasmacytic infiltrate; 20 of them were H.pylori (+), 23 cases showing atrophy; 20 of them were H.pylori (+) and the remaining 3 cases were H.pylori (-); they were grade 1 . Intestinal metaplasia were seen in 16 cases; 15 of them were H.pylori (+). Glandular dysplasia was detected only in 10 cases; all of them were H.pylori (+). The degree of activity of chronic gastritis was summarized in table (1). 


\begin{tabular}{|c|c|c|c|c|c|c|c|c|c|c|c|c|c|c|c|c|c|c|c|c|c|}
\hline & \multicolumn{4}{|c|}{$\begin{array}{l}\text { PNLS } \\
n=38\end{array}$} & \multicolumn{4}{|c|}{$\begin{array}{l}\text { Lymphocytes } \\
\qquad n=26\end{array}$} & \multicolumn{4}{|c|}{$\begin{array}{l}\text { Atrophy } \\
n=23\end{array}$} & \multicolumn{4}{|c|}{$\begin{array}{c}\mathrm{IM} \\
\mathrm{N}=16\end{array}$} & \multicolumn{4}{|c|}{$\begin{array}{l}\text { Dysplasia } \\
\qquad n=10\end{array}$} & \multirow[t]{2}{*}{ Sig. } \\
\hline & 0 & 1 & 2 & 3 & 0 & 1 & 2 & 3 & 0 & 1 & 2 & 3 & 0 & 1 & 2 & 3 & 0 & 1 & 2 & 3 & \\
\hline 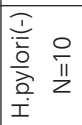 & $\begin{array}{l}0 \\
\circ \\
8 \\
0 \\
0 \\
0\end{array}$ & 0 & 0 & 0 & 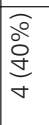 & $\begin{array}{l}\text { ò } \\
\stackrel{\circ}{c} \\
-\end{array}$ & 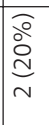 & 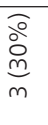 & 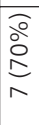 & $\begin{array}{l}\widehat{\partial} \\
\circ \\
0 \\
m \\
m\end{array}$ & 0 & 0 & 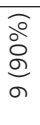 & 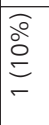 & 0 & 0 & 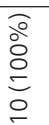 & 0 & 0 & 0 & $\begin{array}{l}\text { mo } \\
0 \\
0\end{array}$ \\
\hline 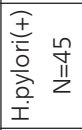 & 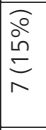 & 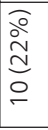 & 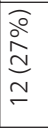 & $\begin{array}{l}\text { ôे } \\
\dot{0} \\
m \\
6\end{array}$ &  & 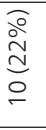 & 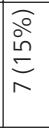 & 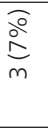 & 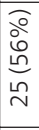 & $\begin{array}{l}a \\
o \\
d \\
\vdots \\
\vdots \\
\vdots\end{array}$ & $\begin{array}{l}\widehat{\Im} \\
\stackrel{\infty}{\infty} \\
\infty\end{array}$ & $\begin{array}{l}\stackrel{a}{\circ} \\
\stackrel{v}{-}\end{array}$ & 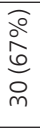 & 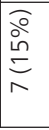 & $\begin{array}{l}\frac{\partial}{\partial} \\
\stackrel{m}{c} \\
\sigma\end{array}$ & 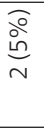 & $\begin{array}{l}\frac{a}{2} \\
\stackrel{2}{N} \\
\ln \\
m\end{array}$ & 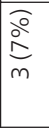 & 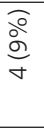 & 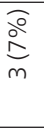 & $\begin{array}{l}\dot{J} \\
\text { Oे }\end{array}$ \\
\hline Sig & \multicolumn{4}{|c|}{0.001} & \multicolumn{4}{|c|}{0.03} & \multicolumn{4}{|c|}{0.04} & \multicolumn{4}{|c|}{0.01} & \multicolumn{4}{|c|}{0.02} & \\
\hline
\end{tabular}

Table 1. The activity of chronic gastritis in the studied cases

Table (2) summarize the correlation study between the histological variables and the studied oncogens. According to p53, the positive cases were 12 and all of them were H.pylori (+), 4 of them were,+ 5 were ++ and 3 were +++ as in figure (1). There were a significant difference between the staining positivity of p53 and all the studied variables including neutrophilic infiltrate, lymphoplasmacytic infiltrate, gastric glandular atrophy, intestinal metaplasia and dysplasia. According to c-Myc staining, the total positive cases were 10 and all of them were H.pylori (+) cases, 4 were,+ 3 were ++ as seen in figure (2) and 3 were +++ . And again there were a significant correlation between the staining positivity and the grades of the studied variables. As the grade of chronic gastritis activity increases, the expression positivity of both p53 and cMyc increases, with significant difference between the grades as seen in table (2).

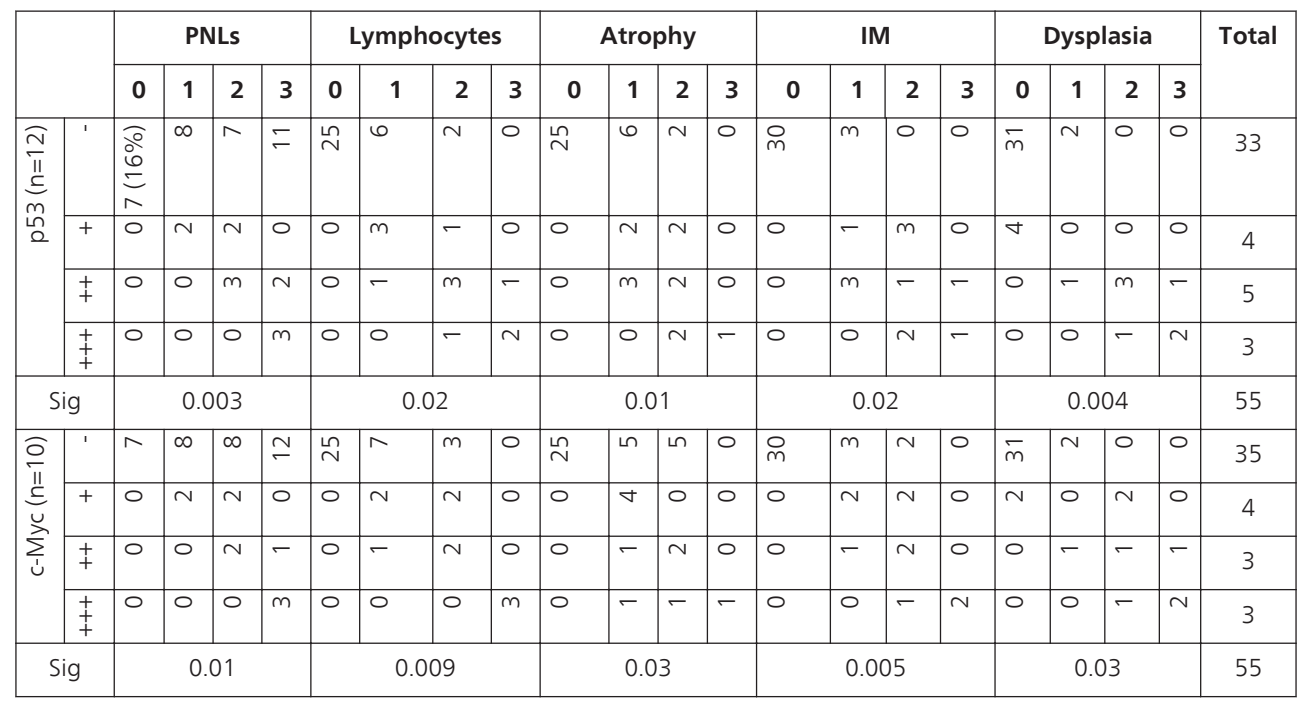

Table 2. Correlation between the p53 and c-Myc and the studied histological variables in H.pylori (+) cases 


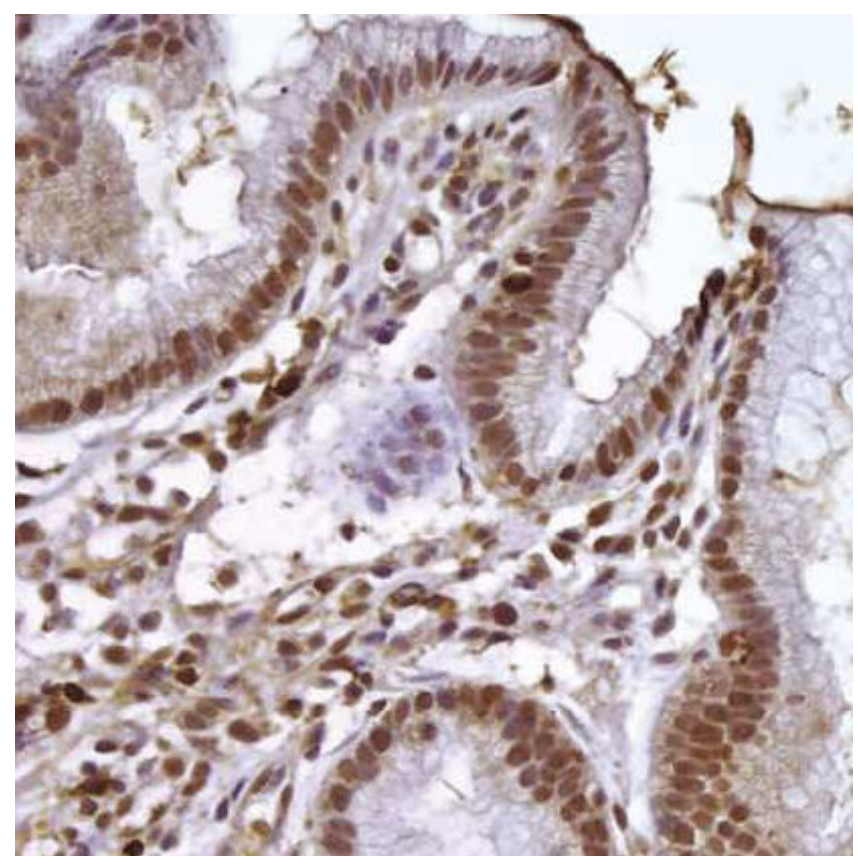

Figure 1. H.pylori (+) case showing +++ p53 expression, this case showed moderate intestinal metaplasia, note the intense inflammatory infiltrate. (strept avidin-biotin-DAB, 400x).

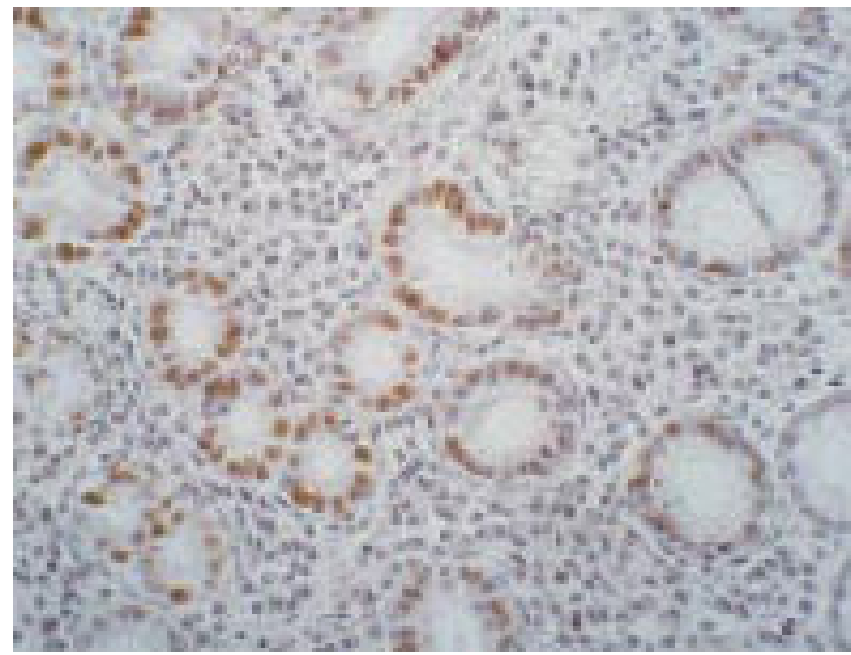

Figure 2. c-Myc moderate expression in a case of H.pylori (+) case, the positivity mainly in intestinal metaplasia portion. (strept avidin-biotin-DAB, 200x). 
After treatment and complete resolution of H.pylori infection in all the cases, the same histopathological variables and immunohistochemical stainig of the studied oncogenes were evalualed. The activity of chronic gastritis was measured and summarized in table (3). It was found that the number of the cases which were positive for neutrophilic infiltrate, lymphocytic infiltrtate, atrophy, intestinal metaplsia and even the glandular dysplasia were less than the number before the treatment and even the grading of all these parameters become much less as shown in table (3) when compared with table (1) with significant difference between the same cases before and after treatment.

\begin{tabular}{|c|c|c|c|c|c|c|c|c|c|c|c|c|c|c|c|c|c|c|c|c|}
\hline & \multicolumn{4}{|c|}{ PNLS } & \multicolumn{4}{|c|}{ Lymphocytes } & \multicolumn{4}{|c|}{ Atrophy } & \multicolumn{4}{|c|}{ IM } & \multicolumn{4}{|c|}{ Dysplasia } \\
\hline & \multicolumn{4}{|c|}{$\mathrm{n}=18$} & \multicolumn{4}{|c|}{$n=8$} & \multicolumn{4}{|c|}{$n=9$} & \multicolumn{4}{|c|}{$n=7$} & \multicolumn{4}{|c|}{$n=7$} \\
\hline & 0 & 1 & 2 & 3 & 0 & 1 & 2 & 3 & 0 & 1 & 2 & 3 & 0 & 1 & 2 & 3 & 0 & 1 & 2 & 3 \\
\hline 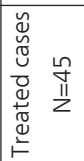 & 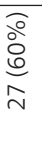 & $\begin{array}{l}\widehat{o} \\
\stackrel{o}{m} \\
m \\
\stackrel{m}{\sim}\end{array}$ & $\begin{array}{l}\widehat{O} \\
\stackrel{\partial}{\Sigma} \\
m\end{array}$ & 0 & $\begin{array}{l}\widehat{o} \\
\grave{\infty} \\
\infty \\
\hat{m}\end{array}$ & $\begin{array}{l}\widehat{\widehat{o}} \\
\stackrel{\sigma}{\sigma}\end{array}$ & $\frac{\widehat{\partial}}{\stackrel{\partial}{n}}$ & 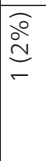 & $\begin{array}{l}\widehat{\partial} \\
\grave{0} \\
\infty \\
6 \\
m\end{array}$ & 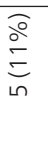 & $\begin{array}{l}\widehat{\partial} \\
\text { oे } \\
\text { वे }\end{array}$ & 0 & $\begin{array}{l}\widehat{\supset} \\
\stackrel{0}{\infty} \\
\infty \\
\infty \\
m\end{array}$ & $\begin{array}{l}\stackrel{\widehat{\partial}}{\stackrel{D}{n}} \\
\mathrm{~m}\end{array}$ & $\begin{array}{l}\frac{\partial}{\partial} \\
\frac{1}{m}\end{array}$ &  & $\begin{array}{l}\widehat{\partial} \\
\partial \\
\Phi \\
\infty \\
\infty \\
m\end{array}$ & $\begin{array}{l}\widehat{\partial} \\
\stackrel{D}{D} \\
m\end{array}$ & $\frac{\widehat{o}}{\stackrel{\partial}{d}}$ & $\begin{array}{l}\frac{\partial}{\partial} \\
\stackrel{d}{d} \\
\text { d }\end{array}$ \\
\hline Sig & \multicolumn{4}{|c|}{0.004} & \multicolumn{4}{|c|}{0.003} & \multicolumn{4}{|c|}{0.005} & \multicolumn{4}{|c|}{0.009} & \multicolumn{4}{|c|}{0.007} \\
\hline
\end{tabular}

Table 3. The activity of chronic gastritis in the studied cases after treatment:

As regarding p53 study after treatment, the number of positive cases become only 7 instead of 12,3 cases were,+ 3 cases were ++ as seen in figure (3) and only one case was +++ . as regarding the correlative study between p53 and the histological variables, there still positive correlation between the p53 and all the variables of chronic gastritis as summarized in table (4).

\begin{tabular}{|c|c|c|c|c|c|c|c|c|c|c|c|c|c|c|c|c|c|c|c|c|c|c|}
\hline & & \multicolumn{4}{|c|}{ PNLs } & \multicolumn{4}{|c|}{ Lymphocytes } & \multicolumn{4}{|c|}{ Atrophy } & \multicolumn{4}{|l|}{ IM } & \multicolumn{4}{|c|}{ Dysplasia } & \multirow[t]{2}{*}{ Total } \\
\hline & & 0 & 1 & 2 & 3 & 0 & 1 & 2 & 3 & 0 & 1 & 2 & 3 & 0 & 1 & 2 & 3 & 0 & 1 & 2 & 3 & \\
\hline \multirow{4}{*}{ 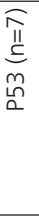 } & 1 & $\grave{v}$ & $\mp$ & 0 & 0 & $\hat{m}$ & - & 0 & 0 & $\ddot{m}$ & $N$ & 0 & 0 & $\infty$ & 0 & 0 & 0 & $\stackrel{\infty}{m}$ & 0 & 0 & 0 & 38 \\
\hline & + & 0 & $m$ & 0 & 0 & 0 & $\sim$ & - & 0 & 0 & $N$ & - & 0 & 0 & $\sim$ & 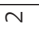 & 0 & 0 & $N$ & - & 0 & 3 \\
\hline & + & 0 & - & $\sim$ & 0 & 0 & - & $\sim$ & 0 & 0 & - & $\sim$ & 0 & 0 & - & - & 0 & 0 & - & - & - & 3 \\
\hline & + & 0 & 0 & - & 0 & 0 & 0 & 0 & - & 0 & 0 & $\tau$ & 0 & 0 & 0 & 0 & - & 0 & 0 & 0 & - & 1 \\
\hline \multicolumn{2}{|c|}{ Sig } & \multicolumn{4}{|c|}{0.01} & \multicolumn{4}{|c|}{0.01} & \multicolumn{4}{|c|}{0.03} & \multicolumn{4}{|c|}{0.02} & \multicolumn{4}{|c|}{0.01} & 45 \\
\hline \multirow{4}{*}{ 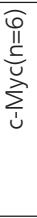 } & 1 & ปิ & $\mp$ & - & 0 & $\hat{m}$ & - & - & 0 & $\ddot{m}$ & $m$ & 0 & 0 & $\hat{m}$ & - & - & 0 & $\stackrel{\infty}{m}$ & - & 0 & 0 & 39 \\
\hline & + & 0 & $N$ & - & 0 & 0 & $m$ & 0 & 0 & 0 & $\sim$ & - & 0 & - & - & - & 0 & 0 & $\sim$ & - & 0 & 3 \\
\hline & + & 0 & - & - & 0 & 0 & 0 & $\sim$ & 0 & 0 & 0 & $\sim$ & 0 & 0 & - & - & 0 & 0 & 0 & - & - & 2 \\
\hline & + & 0 & - & 0 & 0 & 0 & 0 & 0 & - & 0 & 0 & - & 0 & 0 & 0 & 0 & - & 0 & 0 & 0 & - & 1 \\
\hline \multicolumn{2}{|c|}{ Sig } & \multicolumn{4}{|c|}{0.03} & \multicolumn{4}{|c|}{0.04} & \multicolumn{4}{|c|}{0.04} & \multicolumn{4}{|c|}{0.02} & \multicolumn{4}{|c|}{0.01} & 45 \\
\hline
\end{tabular}

Table 4. Correlation between p53 and c-Myc and the studied histological variables after treatment 


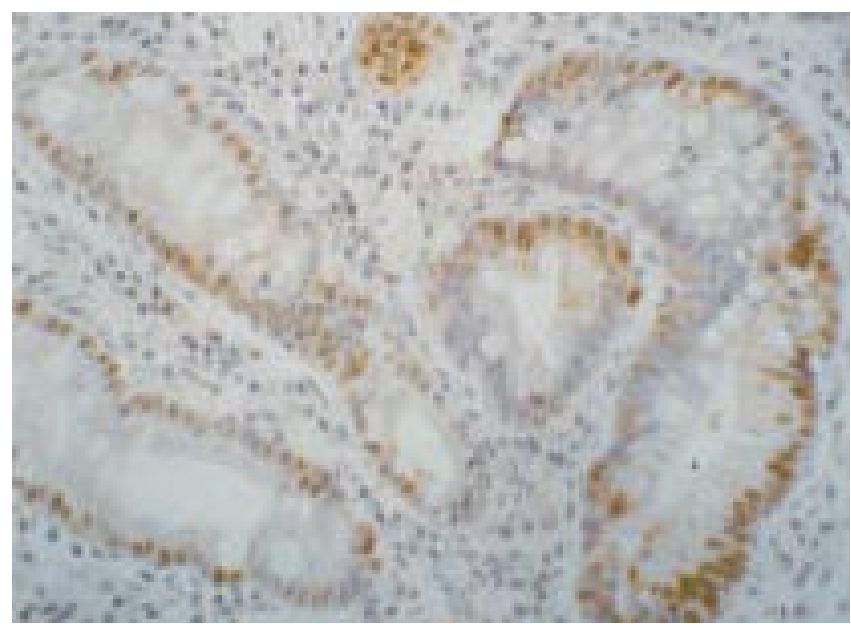

Figure 3. p53 expression after treatment in a case of chronic gastritis was +++ and become ++ confined to the area of intestinal metasplasia (strept avidin-biotin-DAB, 400x).

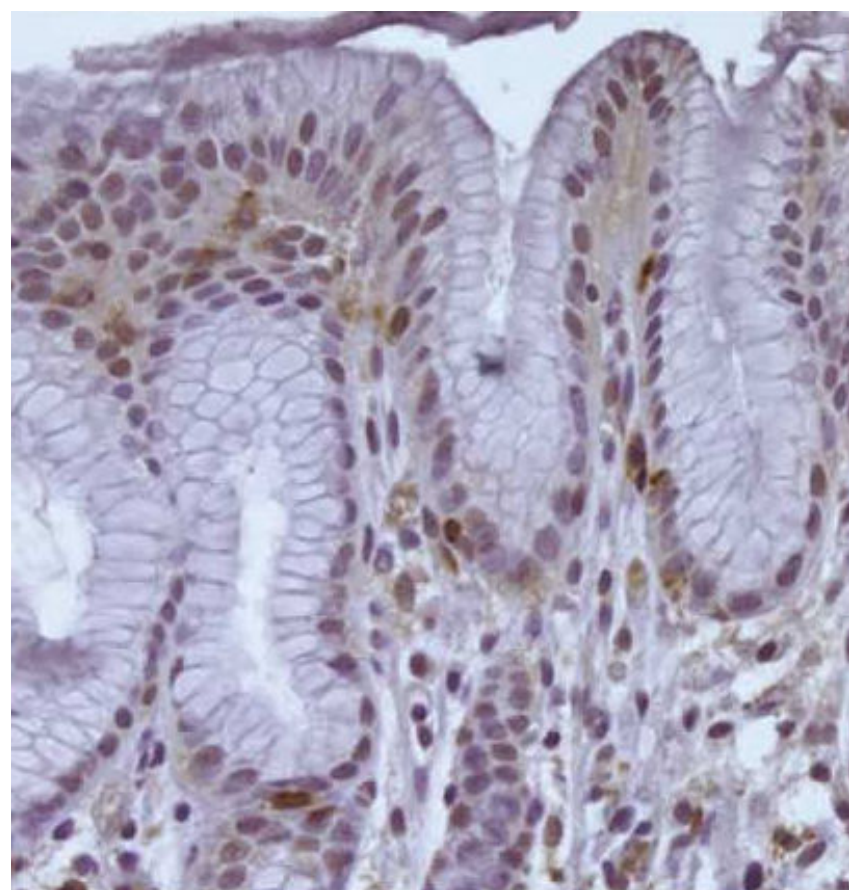

Figure 4. c-Myc expression after treatment was ++ and become + expression only. (strept avidin-biotin-DAB, 400x). 
On the other hand, as regarding c-Myc study after treatment, the number of positive cases become only 6 instead of 10, 3 cases were + as seen in figure (4), 2 cases were ++ and only one case was +++. as regarding the correlative study between c-Myc positivity and the histological variables, there still positive correlation between the c-Myc and all the variables of chronic gastritis as summarized in table (4).

\section{Discussion}

In the present study, it was found that large number of chronic gastritis cases were associated with H.pylori. In H.pylori (+) cases, the majority showed active neutrophilic infiltrate, lymphocytic infiltrate with occasional lymphoid follicles, atrophy, intestinal metaplasia and dysplasia.

c-Myc and p53 expression are the most widely used markers of genomic instability [10]. Interestingly, in this work, c-Myc and p53 expression were not detected in H.pylori (-) cases and this was similar to Van Der et al study [11].

In this work, with the lesions changed between the studied cases from active chronic gastritis to atrophic gastritis to intestinal metaplasia to dysplasia, the positive immunostaining grades of p53 expression increased significantly, the increasing tendency for p53 had a good linear correlation with lesions progression toword the more dangerous precancerous lesions. It is indicated that increased p53 expression may be important molecular events involved in the early stage of gastric carcinogenesis. Thus, this accumulation may be useful biomarkers to assess risk for the development of gastric carcinoma. This was similar to other authors $[1,4,12]$ who have reported that H.pylori-positive gastritis especially that accompanied with intestinal metaplasia showed a higher p53 expression. But unlike Anagnostopoulos et al [13] who reported that p53 was expressed only in cases with high grade dysplasia and not in pre-dysplastic stages and concluded that p53 mutation is a late event during the development of gastric cancer. Our results confirm the relationship between atrophic and/or metaplastic gastric mucosa with H.pylori infection and p53 expression.

Marinone et al [14] data indicated that irreversible genetic changes in the p53 protein has not yet occurred in non-neoplastic gastric mucosa with metaplasia and H.pylori related chronic gastritis and they concluded that the increase in p53 levels is due to an increased production of the wild-type protein probably related to an inflammatory response induced by H.pylori infection.

In this work, H.pylori (-) cases were completely negative for c-Myc expression while H.pylori infection was associated with positive expression of c-Myc unlike others [15] who found that no evidence of expression of c-Myc in any gastritis sample. c-Myc has been reported to be increased in H.pylori-associated gastritis, is associated with increased cell proliferation.

The results of this study may be explained by other studies [6,7] who found that in H.pyloriassociated gastritis, there is downregulation of p27 and increased c-Myc, the net result of 
which is increased cell proliferation and also H.pylori may influence both telomerase activity and c-Myc expression in chronic atrophic gastritis.

Again in Zhan et al [16] study, expression of c-Myc was significantly higher in carcinoma than that in dysplasia than in metaplasia. Also the expression of c-Myc in metaplastic cases and dysplasia with H.pylori infection was significantly higher than that without infection; in this work, c-Myc was completely negative in cases without H.pylori infection. So this study support the others who concluded that H.pylori infection can cause serious imbalance between cell proliferation and apoptosis in the precancerous lesions, giving chances for gastric carcinogenesis.

After eradication treatment, it was found that there were decrease in the cases showed neutrophilic and lymphocytic infiltration and even in the same case there was decrease in the grade of the inflammatory infiltrate. Also the number and the grade of atrophy, intestinal metaplasia and dysplasia were also significantly decreased.

The effects of H.pylori eradication on atrophy and intestinal metaplasia are controversial in the previous studies. Some authors $[17,18]$ have reported similar results with the present work. The patchy nature of the lesions and the subjective nature of the interpretation may account for these controversial findings. Our finding of disappearance or regression of atrophy after H.pylori eradication suggests that inflammatory infiltration plays a role in this controversial histological finding. On the other hand, some studies [11] revealed no changes in intestinal metaplasia and atrophy were detected after H.pylori eradication. While others [19] reported that H.pylori eradication does not reduce the histologic metaplasia score, but changes the cellular phenotype of metaplasia. This change of phenotype may be an important factor in the reduction of cancer incidence after eradication of H.pylori.

In the present study, H.pylori eradication led to a significant reduction in the expression of p53. The number of p53-positive patients was significantly decreased and the grade of positivity was also deceased significantly. Masaaki et al [1] also described a reduction in the expression of p53 after eradication. They reported that H.pylori eradication reduced gastritis activity, atrophy, and complete metaplasia, accompanied by the disappearance of genomic instability markers.

After eradication therapy, the number of cases and the grade of positive c-Myc expression was significantly deceased. This results was the same of others [7] who concuded that H.pylori downregulates p27 and this is reversed following H.pylori eradication.

Finally we can conclude that, H.pylori infection in the gastric mucosa may be implicated in the pathway of gastric carcinogenesis. it seems to be responsible for genomic instability and its eradication can reverse inflammation and related atrophy, metaplasia, and genomic instability. So, we recommend that H.pylori patients especially those with precancerous lesions should receive intense eradication therapy and closely monitored. Because this study confirm that eradication reverses the atrophic changes in the gastric mucosa and the genetic instability, thus may prevent the development of gastric cancer 


\section{Author details}

Hanan AlSaeid Alshenawy ${ }^{1 *}$ and Amr Mahrous Alshafey ${ }^{2}$

*Address all correspondence to: hanan_alshenawy@yahoo.com

1 Department of Pathology, Faculty of Medicine, Tanta University, Tanta, Egypt

2 Department of Internal Medicine, Faculty of Medicine, Tanta University, Tanta, Egypt

\section{References}

[1] Masaaki K, Kazunari M, Tadayoshi O, et al., Expression of mutant type-p53 products in $\mathrm{H}$ pylori-associated chronic gastritis. World J Gastroenterol, 2007, 14;13(10): 1541-1546.

[2] Zhu Y, Shu X, Chen J, et al., Effect of Helicobacter pylori eradication on oncogenes and cell proliferation. Eur J Clin Invest. 2008 ;38 (9):628-633.

[3] Nardone G, Staibano S, Rocco A, et al., Effect of Helicobacter pylori infection and its eradication on cell proliferation, DNA status, and oncogene expression in patients with chronic gastritis. Gut 1999;44:789-799

[4] Chang W, Li Dong W, Lian Hua J, et al., Expression of p53, inducible nitric oxide synthase and vascular endothelial growth factor in gastric precancerous and cancerous lesions: correlation with clinical features. BMC Cancer. 2002; 2-8.

[5] James G., Barbara J, Charles A. et al., Germ-Line p53-targeted Disruption Inhibits Helicobacter-induced Premalignant. Lesions and Invasive Gastric Carcinoma through Down-Regulation of Th1 Proinflammatory Responses. Cancer Research. 2002,(1), 62, 696-702.

[6] Zhang G, Gu Y, Zhao Z, et al., Coordinate increase of telomerase activity and c-Myc expression in Helicobacter pylori-associated gastric diseases. World J Gastroenterol. 2004,15;10(12):1759-1762.

[7] Kim S S, Meitner P, Konkin T, et al., Altered expression of Skp2, c-Myc and p27 proteins but not mRNA after $\mathrm{H}$. pylori eradication in chronic gastritis. Modern Pathology. 2006, 19, 49-58.

[8] Lan J, Xiong Y, Lin $Y$, et al., Helicobacter pylori infection generated gastric cancer through $\mathrm{p} 53-\mathrm{Rb}$ tumor-suppressor system mutation and telomerase reactivation. World J Gastroenterol. 2003; 9 (1):54-58.

[9] Dixon M, Genta R, Yardley J, et al., Classification and grading of gastritis. The updated Sydney System. International Workshop on the Histopathology of Gastritis, Houston 1994. Am J Surg Pathol. 1996; 20: 1161-1181. 
[10] Liang-jing W, Shu-jie C, Zhe C, et al, Morphological and pathologic changes of experimental chronic atrophic gastritis (CAG) and the regulating mechanism of protein expression in rats. J Zhejiang Univ Sci B. 2006; 7(8): 634-640.

[11] Van Der H, Van Der E, Dekker F, et al. Effect of Helicobacter pylori eradication on gastritis in relation to CagA: a prospective 1-year follow-up study. Gastroenterology 1997;113:25-30.

[12] Unger Z, Molnar B, Pronai L, et al., Mutant p53 expression and apoptotic activity of Helicobacter pylori positive and negative gastritis in correlation with the presence of intestinal metaplasia. Eur J Gastroenterol Hepatol 2003; 15: 389-393.

[13] Anagnostopoulos G, Stefanou D, Arkoumani E, et al., Immunohistochemical expression of cell-cycle proteins in gastric precancerous lesions. 2008;23 (4):626-631.

[14] Marinone C, Martinetti A, Mestriner M, et al., p53 evaluation in gastric mucosa of patients with chronic Helicobacter pylori infection. Anticancer Res. 2001;21(2A): 1115-1118.

[15] Wang J, Chi D, Kalin G, et al., Helicobacter pylori infection and oncogene expressions in gastric carcinoma and its precursor lesions. Dig Dis Sci. 2002;47(1):107-113.

[16] Zhan N, Xiong Y, Lan J, et al., Relationship between Helicobacter pylori infection and expression of c-Myc, Bcl-2, and Bax protein in different gastric mucosa lesions. Ai Zheng. 2003;22(10):1034-1037.

[17] Pasztorova I, Chinyama C, Filipe M, et al., Regression of gastric intestinal metaplasia after eradication of Helicobacter pylori: a prospective study [abstract]. Gut 1996; 39(suppl 2):A55.

[18] Murakami K, Kodama M, Sato R, et al., Helicobacter pylori eradication and associated changes in the gastric mucosa. Expert Rev Anti Infect Ther 2005; 3: 757-764.

[19] Watari J, Das K, Amenta P, et al., Effect of eradication of Helicobacter pylori on the histology and cellular phenotype of gastric intestinal metaplasia. Clin Gastroenterol Hepatol. 2008;6(4):409-417. 
